\title{
Coulomb interaction in the spin Hall effect
}

\author{
Liangbin $\mathrm{Hu}, \mathrm{Ju} \mathrm{Gao}$, and Shun-Qing Shen \\ Department of Physics, The University of Hong Kong, Pokfulam Road, Hong Kong, China
}

(Received 9 June 2003; published 9 October 2003)

\begin{abstract}
The influence of the electronic Coulomb interaction on the spin-orbit-coupling induced spin Hall effect was discussed. It was shown that the electronic Coulomb interaction may decrease substantially the spin-orbitcoupling induced transverse spin accumulation in a sample when a longitudinal charge current circulates in it, and the decrease of the transverse spin accumulation due to the influences of the Coulomb interaction is determined by the ratio of the spin-drag resistivity to the ordinary resistivity of the sample.
\end{abstract}

DOI: 10.1103/PhysRevB.68.153303

The spin Hall effect is a transverse spin accumulation effect induced by the spin-orbit coupling (SOC) of moving conduction electrons when a longitudinal charge current circulates in a thin slab of nonmagnetic or ferromagnetic metals (or semiconductors). ${ }^{1,2}$ The spin Hall effect is very different from the spin accumulation effect found in magnetic multilayers when a charge current flows in the direction perpendicular to the plane of the layers. ${ }^{3-6}$ From theoretical viewpoints, the spin accumulation effect found in magnetic multilayers is caused by the discontinuity of material properties at the interfaces of alternating layers, but the spin Hall effect arises from the SOC induced left-right asymmetric scattering of moving conduction electrons and can occur only in thin slab geometry. The SOC induced left-right asymmetric scattering was known as skew scattering in the literature, and was believed to be the origin of the anomalous Hall coefficients found in ferromagnetic metals. ${ }^{7,8}$ In the spin Hall effect, as illustrated in Fig. 1, when a longitudinal charge current flows in a thin slab of paramagnetic or ferromagnetic metals (or semiconductors), due to the SOC induced leftright asymmetric scattering, spin-up electrons will have a larger probability to be scattered to the right and spin-down electron will have a larger probability to be scattered to the left, leading to transverse nonequilibrium spin accumulation at both sides of the slab. ${ }^{1,2}$ It is anticipated that this effect may find some practical applications in the emerging field of spintronics. ${ }^{1,9,10}$ For instance, this effect can be applied to generate and/or measure a pure spin current, which carry only spins but carry no charges. ${ }^{1}$ In this paper we discuss the influences of the electronic Coulomb interaction on the spin Hall effect. This is an interesting question about the spin Hall effect. Since no charge imbalance occurs in the spin Hall effect, at first sight it seems that the electronic Coulomb interaction should have no substantial influence on the spin Hall effect. However, in this paper we will show that this is not the case. We will show that the electronic Coulomb interaction may have some significant influence on the spin Hall effect. The reason for this is that in the spin Hall effect, due to the spin-orbit coupling of moving conduction electrons, a transverse relative movement will be caused between the spin-up and -down components, but arising from the electronic Coulomb interaction, there will inherently exist a friction between the relative movements of spin-up and -down components. ${ }^{11}$ In this paper we will show that due to this inherent friction, the spin Hall effect may be decreased substantially by the electronic Coulomb interaction.
PACS number(s): 72.15.Gd, 73.50.Jt, 72.10.-d

The system is described schematically in Fig. 1. We will assume that the length of the slab is much larger than its width $L$, so that in the longitudinal direction the current density is uniform. The thickness of the slab is assumed to be much smaller than the spin-diffusion length so that the spin current perpendicular to the film can be neglected. To calculate the SOC induced transverse spin current, we start from the Boltzman equation, which reads

$$
\frac{\hbar \vec{k}}{m} \cdot \nabla_{r} f^{\sigma}(\vec{k}, \vec{r})-e \vec{E}_{e x t} \cdot \frac{\hbar \vec{k}}{m} \frac{\partial f^{0}\left(\epsilon_{k \sigma}\right)}{\partial \epsilon_{k \sigma}}=-\left(\frac{\partial f^{\sigma}}{\partial t}\right)_{\text {coll. }},
$$

where $\vec{E}_{\text {ext }}=E_{x} \vec{e}_{x}$ is the longitudinal external electric field, $f^{\sigma}(\vec{k}, \vec{r})$ and $f^{0}\left(\epsilon_{k \sigma}\right)$ are the total and equilibrium distribution functions of conduction electrons with spin $\sigma$, respectively, and as usual, $f^{0}\left(\epsilon_{k \sigma}\right) \equiv 1 /\left(e^{\beta\left(\epsilon_{k \sigma}-E_{F}\right)}+1\right)$, where $E_{F}$ denotes the chemical potential in the equilibrium state, $\beta \equiv 1 / k_{B} T$ (i.e., the inverse of the product of the Boltzman constant and the temperature), $\epsilon_{\vec{k} \sigma} \equiv \hbar^{2} k^{2} / 2 m+\epsilon_{\sigma}^{(0)}$, and $\epsilon_{\sigma}^{(0)}$ denotes the band offset. The collision term $\left(\partial f^{\sigma} / \partial t\right)_{\text {coll. }}$ can be divided into three parts, which arise from the non-spin-flip and spinflip electron-impurity scattering processes and the electronelectron scattering process, respectively. The contribution arising from the electron-electron scattering process is given by

$$
\begin{aligned}
\left(\frac{\partial f^{\sigma}(\vec{k}, \vec{r})}{\partial t}\right)^{(e-e)} & \\
= & -\sum_{\beta, \sigma^{\prime}, \beta^{\prime}} \int \frac{d^{3} \vec{k}^{\prime} d^{3} \vec{p} d^{3} \vec{p}^{\prime}}{(2 \pi)^{3 \times 3}} w^{(e-e)} \\
& \times\left(\vec{k} \sigma, \vec{p} \beta ; \vec{k}^{\prime} \sigma^{\prime}, \vec{p}^{\prime} \beta^{\prime}\right)\left\{f^{\sigma}(\vec{k}, \vec{r}) f^{\beta}(\vec{p}, \vec{r})\right. \\
& \times\left[1-f^{\sigma^{\prime}}\left(\vec{k}^{\prime}, \vec{r}\right)\right]\left[1-f^{\beta^{\prime}}\left(\vec{p}^{\prime}, \vec{r}\right)\right] \\
& \left.\left.-\left[1-f^{\sigma}(\vec{k}, \vec{r})\right]\left[1-f^{\beta}(\vec{p}, \vec{r})\right] f^{\sigma^{\prime}}\left(\vec{k}^{\prime}, \vec{r}\right) f^{\beta^{\prime}}\left(\vec{p}^{\prime}, \vec{r}\right)\right]\right\} \\
& \times \delta\left(\epsilon_{\vec{k} \sigma}+\epsilon_{\vec{p} \beta}-\epsilon_{\vec{k}^{\prime} \sigma^{\prime}}-\epsilon_{\vec{p}^{\prime} \beta^{\prime}}\right) \delta\left(\vec{k}+\vec{p}-\vec{k}^{\prime}-\vec{p}^{\prime}\right),
\end{aligned}
$$

where $w^{(e-e)}\left(\vec{k} \sigma, \vec{p} \beta ; \vec{k}^{\prime} \sigma^{\prime}, \vec{p}^{\prime} \beta^{\prime}\right)$ is the probability of the electron-electron scattering process $(\vec{k} \sigma, \vec{p} \beta)$ $\rightarrow\left(\vec{k}^{\prime} \sigma^{\prime}, \vec{p}^{\prime} \beta^{\prime}\right)$. The contributions from the non-spin-flip and spin-flip electron-impurity scattering processes are given by 


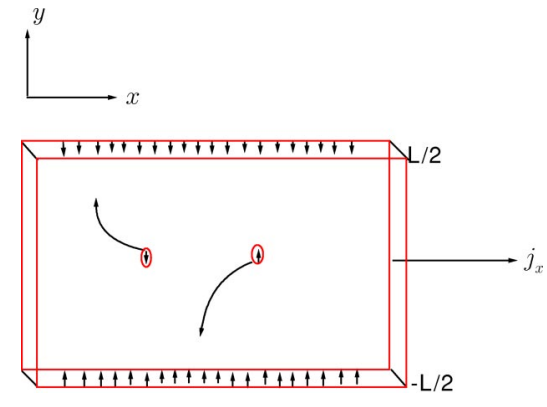

FIG. 1. Illustration of the spin Hall effect. When a longitudinal charge current flows in a thin slab of paramagnetic or ferromagnetic metals (or semiconductors), the spin-orbit-coupling of moving conduction electrons will cause transverse nonequilibrium spin accumulation at both sides of the slab. (The two boundaries of the slab are assumed to be located at $y= \pm L / 2$.)

$$
\begin{aligned}
\left(\frac{\partial f^{\sigma}(\vec{k}, \vec{r})}{\partial t}\right)_{\sigma, \sigma}^{(i)}= & -\int \frac{d^{3} \vec{k}^{\prime}}{(2 \pi)^{3}} w_{\sigma, \sigma}^{(i)}\left(\vec{k}, \vec{k}^{\prime}\right) \delta\left(\epsilon_{\vec{k} \sigma}-\epsilon_{\vec{k}^{\prime} \sigma}\right) \\
& \times\left[f^{\sigma}(\vec{k}, \vec{r})-f^{\sigma}\left(\vec{k}^{\prime}, \vec{r}\right)\right], \\
\left(\frac{\partial f^{\sigma}(\vec{k}, \vec{r})}{\partial t}\right)_{\sigma,-\sigma}^{(i)}= & -\int \frac{d^{3} \vec{k}^{\prime}}{(2 \pi)^{3}} w_{\sigma,-\sigma}^{(i)}\left(\vec{k}, \vec{k}^{\prime}\right) \delta\left(\epsilon_{\vec{k} \sigma}-\epsilon_{\vec{k}^{\prime},-\sigma}\right) \\
& \times\left[f^{\sigma}(\vec{k}, \vec{r})-f^{-\sigma}\left(\vec{k}^{\prime}, \vec{r}\right)\right],
\end{aligned}
$$

where $w_{\sigma, \sigma}^{(i)}\left(\vec{k}, \vec{k}^{\prime}\right)$ and $w_{\sigma,-\sigma}^{(i)}\left(\vec{k}, \vec{k}^{\prime}\right)$ are the non-spin-flip and spin-flip electron-impurity scattering rates, respectively. There are two different approaches to take into account the effect of the spin-orbit coupling. ${ }^{2,12,13}$ One approach is to calculate the scattering probabilities $w_{\sigma, \sigma}^{(i)}\left(\vec{k}, \vec{k}^{\prime}\right)$ and $w_{\sigma,-\sigma}^{(i)}\left(\vec{k}, \vec{k}^{\prime}\right)$ first by taking the spin-orbit coupling into account, and then to find the total distribution function $f^{\sigma}$ from Eq. (1). In this approach, $w_{\sigma, \sigma}^{(i)}\left(\vec{k}, \vec{k}^{\prime}\right)$ and $w_{\sigma,-\sigma}^{(i)}\left(\vec{k}, \vec{k}^{\prime}\right)$ will contain skew scattering terms, i.e., asymmetric terms with respect to the incoming and outgoing velocities. The second approach is to find first the total distribution function $f^{\sigma}$ from Eq. (1) by using the scattering probability $w_{\sigma, \sigma}^{(i)}\left(\vec{k}, \vec{k}^{\prime}\right)$ and $w_{\sigma,-\sigma}^{(i)}\left(\vec{k}, \vec{k}^{\prime}\right)$ in the absence of the spin-orbit coupling, and then to include the influence of the spin-orbit coupling by adding an anomalous term in the current, ${ }^{2,12,13}$

$$
\vec{j}^{\sigma}(\vec{r})=\int \frac{d^{3} \vec{k}}{(2 \pi)^{3}}\left[\frac{\hbar \vec{k}}{m}+\vec{\Omega}^{\sigma}(\vec{k})\right] f^{\sigma}(\vec{k}, \vec{r}),
$$

where $\vec{\Omega}^{\sigma}(\vec{k})=\left(\alpha \hbar / \tau^{\sigma}\right) \vec{k} \times \vec{\sigma}$, in which $\alpha$ is the strength of the spin-orbit coupling and $\tau^{\sigma}$ is the non-spin-flip relaxation time of electrons with spin $\sigma$, which will be defined below. It can be shown that this approach is completely equivalent to the first approach up to second order in the spin-orbit coupling constant $\alpha .{ }^{12,13}$ In this paper we will take the second approach. To find the distribution function $f^{\sigma}(\vec{k}, \vec{r})$ in the absence of the spin-orbit-coupling, we assume that the distribution function can be expressed as the sum of the equilibrium and nonequilibrium ones,

$$
\begin{aligned}
f^{\sigma}(\vec{k}, \vec{r})= & f^{0}\left(\epsilon_{\vec{k} \sigma}\right)-e \frac{\partial f^{0}\left(\epsilon_{\vec{k} \sigma}\right)}{\partial \epsilon_{k \sigma}} \mu^{\sigma}(\vec{r}) \\
& +e \tau^{\sigma} \vec{E}^{\sigma}(\vec{r}) \cdot \vec{v} \frac{\partial f^{0}\left(\boldsymbol{\epsilon}_{\vec{k} \sigma}\right)}{\partial \epsilon_{k \sigma}},
\end{aligned}
$$

where $\vec{v}=\hbar \vec{k} / m$ is the velocity of conduction electrons, $\mu^{\sigma}(\vec{r})$ denotes the spin-dependent shift of the chemical potential, which is caused by the SOC induced transverse spin imbalance in the sample, and $\vec{E}^{\sigma}(\vec{r})$ is the effective field felt by moving conduction electrons with spin $\sigma$. Due to the occurrence of the transverse spin imbalance in the sample, in addition to the longitudinal external electric field $E_{x}$, moving conduction electrons will also feel a spin-dependent transverse effective field $E_{y}^{\sigma}$, i.e., $\vec{E}^{\sigma}=E_{x} \vec{e}_{x}+E_{y}^{\sigma} \vec{e}_{y}$. If the influence of the electron-electron interaction is neglected, $E_{y}^{\sigma}$ will be given simply by $E_{y}^{\sigma}=-\partial \mu^{\sigma}(y) / \partial y$, corresponding to the case considered in Refs. 1 and 2. But after taking into account the electronic Coulomb interaction, $E_{y}^{\sigma}$ will depend not only on $\mu^{\sigma}(y)$ but also depend on $\mu^{-\sigma}(y)$; thus $E_{y}^{\sigma}$ cannot be given simply by $E_{y}^{\sigma}=-\partial \mu^{\sigma}(y) / \partial y$. In such a case, the relation between the transverse effective field $E_{y}^{\sigma}$ and the SOC induced transverse spin imbalance in the sample must be derived strictly from Eqs. (1)-(4). This derivation will be given below. Equation (6) is generally valid for an isotropic system slightly perturbed from its equilibrium state. ${ }^{4}$ After substituting Eq. (6) into Eq. (5), the total spin-dependent charge current densities in the sample can be expressed as

$$
\vec{j}^{\sigma}(\vec{r})=C_{\sigma} \vec{E}^{\sigma}(\vec{r})+C_{H}^{\sigma} \vec{E}^{\sigma}(\vec{r}) \times \vec{\sigma},
$$

where $C_{\sigma}=e^{2} \tau^{\sigma}\left(k_{F}^{\sigma}\right)^{3} / 6 \pi^{2} m$ is the ordinary Drude conductivity, and $C_{H}^{\sigma}=e^{2} \alpha\left(k_{F}^{\sigma}\right)^{3} / 6 \pi^{2}$ is the anomalous Hall conductivity caused by the spin-orbit coupling. To obtain the relation between the transverse effective field $E_{y}^{\sigma}$ and the SOC induced transverse spin imbalance in the sample, we substitute Eq. (6) into Eqs. (1)-(4). The left-hand side of Eq. (1) will become

$$
\begin{aligned}
\frac{\hbar \vec{k}}{m} & \cdot \nabla_{r} f^{\sigma}(\vec{k}, \vec{r})-e \vec{E}_{e x t} \cdot \frac{\hbar \vec{k}}{m} \frac{\partial f^{0}\left(\epsilon_{k \sigma}\right)}{\partial \epsilon_{k \sigma}} \\
& =\frac{\hbar \vec{k}}{m} \frac{\partial f^{0}\left(\epsilon_{\vec{k} \sigma}\right)}{\partial \epsilon_{k \sigma}}\left[\frac{\hbar e \tau^{\sigma}}{m} \nabla_{r}\left[\vec{k} \cdot \vec{E}^{\sigma}(\vec{r})\right]-\nabla_{r} \mu^{\sigma}(\vec{r})-e \vec{E}_{\text {ext }}\right],
\end{aligned}
$$

and Eqs. (3) and (4) will become

$$
\begin{aligned}
\left(\frac{\partial f^{\sigma}}{\partial t}\right)_{\sigma, \sigma}^{(i)}=\frac{\hbar e \tau^{\sigma}}{m} \vec{k} \cdot \vec{E}^{\sigma}(\vec{r}) \frac{\partial f^{0}\left(\epsilon_{\vec{k} \sigma}\right)}{\partial \epsilon_{k \sigma}}, \\
\left(\frac{\partial f^{\sigma}}{\partial t}\right)_{\sigma,-\sigma}^{(i)}=\frac{\partial f^{0}\left(\epsilon_{\vec{k} \sigma}\right)}{\partial \epsilon_{k \sigma}} \frac{1}{\tau^{\uparrow, \downarrow}} \\
\times\left[\mu^{\sigma}(\vec{r})-\mu^{-\sigma}(\vec{r})-\frac{\hbar e \tau^{\sigma}}{m} \vec{k} \cdot \vec{E}^{\sigma}(\vec{r})\right],
\end{aligned}
$$


in which $\tau^{\sigma}$ and $\tau^{\uparrow, \downarrow}$ are the impurity induced non-spin-flip and spin-flip electron relaxation times, respectively:

$$
\begin{gathered}
\tau^{\sigma}=\left[\int \frac{d^{3} \vec{k}^{\prime}}{(2 \pi)^{3}} w_{\sigma, \sigma}^{(i)}\left(\vec{k}, \vec{k}^{\prime}\right) \delta\left(\epsilon_{\vec{k} \sigma}-\epsilon_{\vec{k}^{\prime} \sigma}\right)\right]^{-1}, \\
\tau^{\uparrow, \downarrow}=\left[\int \frac{d^{3} \vec{k}^{\prime}}{(2 \pi)^{3}} w_{\uparrow, \downarrow}^{(i)}\left(\vec{k}, \vec{k}^{\prime}\right)\right]^{-1} .
\end{gathered}
$$

As to Eq. (2), it can be shown that within the approximation scheme of Eq. (6), only the scattering process between two electrons with opposite spins will contribute to $\left[\partial f^{\sigma}(\vec{k}, \vec{r}) / \partial t\right]^{(e-e)}$, and the result can be expressed as

$$
\left(\frac{\partial f^{\sigma}}{\partial t}\right)^{(e-e)}=\frac{2 \pi e \beta}{m}\left[\tau^{\sigma} \vec{E}^{\sigma}(\vec{r})-\tau^{-\sigma} \vec{E}^{-\sigma}(\vec{r})\right] \cdot \vec{g}_{\uparrow, \downarrow}(\vec{k}),
$$

where $\vec{g}_{\uparrow, \downarrow}(\vec{k})$ is defined by

$$
\begin{aligned}
\vec{g}_{\uparrow, \downarrow}(\vec{k})= & \int \frac{d^{3} \vec{k}^{\prime} d^{3} \vec{p} d^{3} \vec{p}^{\prime}}{(2 \pi)^{3 \times 3}} w^{(e-e)}\left(\vec{k} \uparrow, \vec{p} \downarrow ; \vec{k}^{\prime} \uparrow, \vec{p}^{\prime} \downarrow\right) \\
& \times\left(\vec{k}-\vec{k}^{\prime}\right) f^{0}\left(\epsilon_{\vec{k} \uparrow}\right) f^{0}\left(\epsilon_{\vec{p} \downarrow}\right)\left[1-f^{0}\left(\epsilon_{\vec{k}^{\prime} \uparrow}\right)\right] \\
& \times\left[1-f^{\sigma}\left(\epsilon_{\vec{p}^{\prime} \downarrow}\right)\right] \delta\left(\epsilon_{\vec{k} \uparrow}+\epsilon_{\vec{p} \downarrow}-\epsilon_{\vec{k}^{\prime} \uparrow}-\epsilon_{\vec{p}^{\prime} \downarrow}\right) \\
& \times \delta\left(\vec{k}+\vec{p}-\vec{k}^{\prime}-\vec{p}^{\prime}\right) .
\end{aligned}
$$

From Eq. (1) and Eqs. (8)-(14), after some straightforward but tedious algebra, the transverse spin-dependent effective field $E_{y}^{\sigma}$ can be expressed in terms of $\partial \mu^{\sigma}(y) / \partial y$ and $\partial \mu^{-\sigma}(y) / \partial y$, and then, from Eq. (7), the SOC induced transverse spin-dependent charge current densities in the sample can be expressed as

$$
j_{y}^{\sigma}=C^{\sigma} E_{y}^{\sigma}-\sigma C_{H}^{\sigma} E_{x},
$$

where $\sigma= \pm 1$, corresponding to the spin-up and -down components, respectively, and

$$
E_{y}^{\sigma}=-b\left(\lambda^{\sigma}\right) \frac{\partial \mu^{\sigma}(y)}{\partial y}-g\left(\lambda^{\sigma}\right) \frac{\partial \mu^{-\sigma}(y)}{\partial y},
$$

where $b\left(\lambda^{\sigma}\right)$ and $g\left(\lambda^{\sigma}\right)$ are defined by

$$
\begin{aligned}
& b\left(\lambda^{\sigma}\right)=\frac{1+\lambda^{\sigma} / \gamma}{\left(1+\lambda^{\sigma} / \gamma\right)^{2}-\left(\lambda^{\sigma}\right)^{2}}, \\
& g\left(\lambda^{\sigma}\right)=\frac{\lambda^{\sigma}}{\left(1+\lambda^{\sigma} / \gamma\right)^{2}-\left(\lambda^{\sigma}\right)^{2}},
\end{aligned}
$$

where $\gamma \equiv n_{\uparrow}^{(0)} / n_{\downarrow}^{(0)}$, i.e., the ratio of the equilibrium densities of the majority and minority electrons, and $\lambda^{\sigma}$ $\equiv R_{\sigma} / R_{D}$, in which $R_{\sigma}=1 / C_{\sigma}$ is the ordinary resistivity of the sample, and $R_{D}$ is a characteristic resistivity arising from the electron-electron scattering process of electrons with opposite spins:

$$
\begin{aligned}
R_{D}= & \frac{\beta}{6 n_{\uparrow}^{(0)} n_{\downarrow}^{(0)} e^{2}} \int \frac{d^{3} \vec{k}}{(2 \pi)^{3}} \int \frac{d^{3} \vec{k}^{\prime}}{(2 \pi)^{3}} \int \frac{d^{3} \vec{p}}{(2 \pi)^{3}} \int \frac{d^{3} \vec{p}^{\prime}}{(2 \pi)^{3}} \\
& \times w^{(e-e)}\left(\vec{k} \uparrow, \vec{p} \downarrow ; \vec{k}^{\prime} \uparrow, \vec{p}^{\prime} \downarrow\right)\left(\vec{k}-\vec{k}^{\prime}\right)^{2} f^{0}\left(\epsilon_{\vec{k} \uparrow}\right) f^{0}\left(\epsilon_{\vec{p} \downarrow}\right) \\
& \times\left[1-f^{0}\left(\epsilon_{\vec{k}^{\prime} \uparrow}\right)\right]\left[1-f^{\sigma}\left(\epsilon_{\vec{p}^{\prime} \downarrow}\right)\right] \delta\left(\epsilon_{\vec{k} \uparrow}+\epsilon_{\vec{p} \downarrow}-\epsilon_{\vec{k}^{\prime} \uparrow}-\epsilon_{\vec{p}^{\prime} \downarrow}\right) \\
& \times \delta\left(\vec{k}+\vec{p}-\vec{k}^{\prime}-\vec{p}^{\prime}\right) .
\end{aligned}
$$

This resistivity characterize the strength of the friction between the relative movement of spin-up and -down components and was called the spin-drag resistivity. ${ }^{11}$ In addition to Eqs. (15)-(18), it can be further shown from Eq. (1) and Eqs. (8)-(14) that the spin-dependent shift of the chemical potential will satisfy the following diffusion equation:

$$
\frac{\partial^{2}}{\partial y^{2}} \mu^{\sigma}(y)=\frac{\mu^{\sigma}(y)-\mu^{-\sigma}(y)}{\left[D\left(\lambda^{\sigma}\right)\right]^{2}},
$$

where $\quad D\left(\lambda^{\sigma}\right)=D_{0}^{\sigma}\left[1+n_{0} \lambda^{\sigma} / n_{\sigma}^{0}\right]^{-1 / 2}$, and $D_{0}^{\sigma}$ $=v_{F}^{\sigma} \sqrt{\tau^{\sigma} \tau^{\uparrow, \downarrow / 3}}$ is the spin-diffusion length in the absence of electron-electron interaction, with $v_{F}^{\sigma}$ denoting the Fermi velocity. From Eq. (20), the transverse spin accumulation in the sample, which is given by $\mu^{\uparrow}(y)-\mu^{\downarrow}(y)$, will satisfy the following equation:

$$
\frac{\partial^{2}}{\partial y^{2}}\left[\mu^{\uparrow}(y)-\mu^{\downarrow}(y)\right]=\frac{\mu^{\uparrow}(y)-\mu^{\downarrow}(y)}{\left[D\left(\lambda^{\uparrow}, \lambda_{\downarrow}\right)\right]^{2}},
$$

where $\left[D\left(\lambda^{\uparrow}, \lambda_{\downarrow}\right)\right]^{-2}=\left[D\left(\lambda^{\uparrow}\right)\right]^{-2}+\left[D\left(\lambda_{\downarrow}\right)\right]^{-2} . D\left(\lambda^{\uparrow}, \lambda_{\downarrow}\right)$ is the renormalized spin-diffusion length in the presence of the electronic Coulomb interaction. From Eq. (21), $\mu^{\uparrow}(y)$ $-\mu^{\downarrow}(y)$ can be expressed as

$$
\mu^{\uparrow}(y)-\mu^{\downarrow}(y)=A e^{y / D\left(\lambda^{\uparrow}, \lambda_{\downarrow}\right)}+B e^{-y / D\left(\lambda^{\uparrow}, \lambda_{\downarrow}\right)},
$$

in which $A$ and $B$ are constant coefficients.

We now apply Eqs. (15)-(18) and Eq. (22) to discuss the SOC induced transverse spin accumulation in the sample. Substituting Eq. (22) into Eq. (15), the transverse spindependent charge current densities in the sample can be expressed as functions of the constant coefficients $A$ and $B$. In the open circuit condition, the transverse spin-dependent charge current densities should satisfy the following condition: $j_{y}^{\sigma}(y)+j_{y}^{-\sigma}(y)=0$ and $j_{y}^{\sigma}( \pm L / 2)=0$. From this condition the coefficients $A$ and $B$ can be determined, and then the transverse spin accumulation in the sample can be obtained, which reads

$$
\begin{aligned}
& \mu^{\uparrow}(y)-\mu^{\downarrow}(y) \\
& =\frac{E_{x} D\left(\lambda^{\uparrow}, \lambda_{\downarrow}\right) \sinh \left[y / D\left(\lambda^{\uparrow}, \lambda_{\downarrow}\right)\right]}{C^{\uparrow} C^{\downarrow}\left[b\left(\lambda^{\uparrow}\right) b\left(\lambda^{\downarrow}\right)-g\left(\lambda^{\uparrow}\right) g\left(\lambda^{\downarrow}\right)\right] \cosh \left[w / 2 D\left(\lambda^{\uparrow}, \lambda_{\downarrow}\right)\right]} \\
& \quad \times\left\{C^{\uparrow} C_{H}^{\downarrow}\left[g\left(\lambda^{\uparrow}\right)-b\left(\lambda^{\uparrow}\right)\right]+C^{\downarrow} C_{H}^{\uparrow}\left[g\left(\lambda^{\downarrow}\right)-b\left(\lambda^{\downarrow}\right)\right]\right\} .
\end{aligned}
$$

From Eq. (23), we observe that after taking into account the electronic Coulomb interaction, the transverse spin accumulation in a sample will have a sensitive dependence on the 


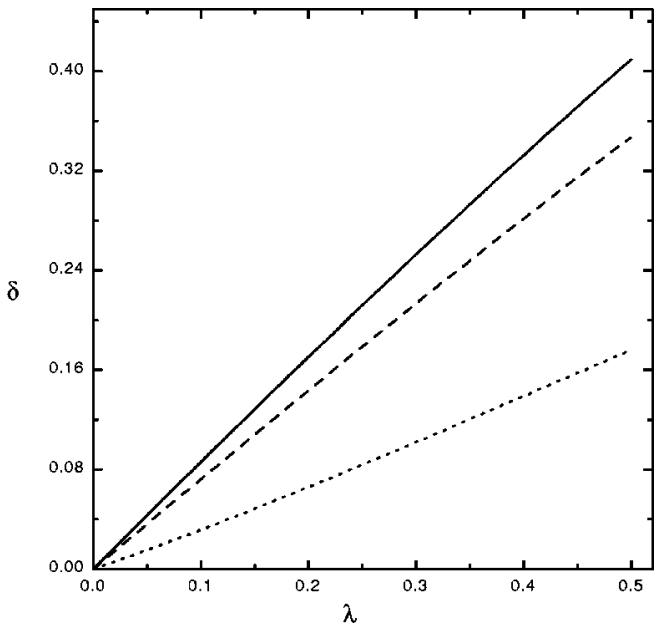

FIG. 2. Illustration of the changes of the ratio $\delta$ with the variation of the ratio $\lambda^{\sigma}$ for three different spin polarization $\gamma$. ( $\gamma=1$ for the dotted line, $\gamma=2$ for the dashed line, and $\gamma=3$ for the solid line. Other parameters used in the calculation have been given in the text.)

ratio of the Coulomb interaction induced spin-drag resistivity to the ordinary resistivity of the sample, indicating that the Coulomb interaction may have some significant influence on the SOC induced transverse spin accumulation in a sample. Since the right-hand side of Eq. (23) is a little complex, the influences of the Coulomb interaction cannot be seen apparently from Eq. (23). To get a qualitative picture of the influences of the Coulomb interaction, one can calculate $\mu^{\uparrow}$ $-\mu^{\downarrow}$ as a function of $\lambda^{\sigma}$ by fixing other parameters in Eq. (23). The change of the transverse spin accumulation in the sample due to the influences of the Coulomb interaction can be characterized by a ratio $\delta \equiv \Delta\left(\mu^{\uparrow}-\mu^{\downarrow}\right) /\left(\mu^{\uparrow}-\mu^{\downarrow}\right)_{\lambda} \sigma_{=0}$, in which $\left(\mu^{\uparrow}-\mu^{\downarrow}\right)_{\lambda^{\sigma}=0}$ is the transverse spin accumulation at the edges of the sample in the absence of the Coulomb interaction and $\Delta\left(\mu^{\uparrow}-\mu^{\downarrow}\right) \equiv\left(\mu^{\uparrow}-\mu^{\downarrow}\right)_{\lambda \sigma=0}-\left(\mu^{\uparrow}\right.$ $\left.-\mu^{\downarrow}\right)_{\lambda^{\sigma \neq 0}}$ is the change of the transverse spin accumulation at the edges of the sample due to the influences of the Coulomb interaction. The typical behavior of the changes of the ratio $\delta$ with the variation of the ratio $\lambda^{\sigma}$ have been plotted in Fig. 2 for three different spin polarization $\gamma .\left(\gamma=n_{\uparrow}^{(0)} / n_{\downarrow}^{(0)}\right.$ $>1$ for a ferromagnetic sample and $\gamma=1$ for a paramagnetic sample.) In plotting Fig. 2 the following parameters were used: the ordinary conductivity $C^{\sigma}=0.1(\mu \Omega \mathrm{cm})^{-1}$, the anomalous Hall conductivity $C_{H}^{\sigma}=10^{-3}(\mu \Omega \mathrm{cm})^{-1}$, the spin-diffusion length $D_{0}=1 \mu \mathrm{m}$, the longitudinal charge current density $j_{x}=10^{6} \mathrm{~A} / \mathrm{cm}^{2}$, and the sample width $L$ $=1 \mu \mathrm{m}$. Such parameters are typical of transition metals. (For simplicity we have assumed that the ordinary conductivity $C^{\sigma}$ and the anomalous Hall conductivity $C_{H}^{\sigma}$ are spin independent, and hence the ratio $\lambda^{\sigma}$ is also spin independent.) For the parameters listed above, the calculated transverse spin accumulation at the edges of the sample will be $5 \mu \mathrm{V}$ if one neglects the Coulomb interaction and it will be decreased from this value after taking into account the Coulomb interaction (i.e., $\lambda^{\sigma} \neq 0$ ). From Fig. 2, one can see that for different spin polarizations $\gamma$, the ratio $\delta$ all increase with the increase of the ratio $\lambda^{\sigma}$, i.e., the stronger the Coulomb interaction is, the larger the decrease of the spin accumulation will be, and it can be noted that compared with the result obtained in the absence of the Coulomb interaction, the decrease of the spin accumulation due to the influences of the Coulomb interaction may be very substantial if the spin-drag resistivity $R_{D}$ is comparable to the ordinary resistivity $R_{\sigma}$ of the sample. In principle, the spin-drag resistivity $R_{D}$ can be calculated from Eq. (6) and as has been shown previously, $R_{D}$ can be as large as a fraction of $R_{\sigma}$, especially for samples with low dimensions. ${ }^{11}$ In such cases the decrease of the spin accumulation due to the influences of the Coulomb interaction can also be as large as a fraction of the corresponding values obtained in the absence of the Coulomb interaction, which can be seen from Fig. 2 .

In conclusion, we have discussed the influence of the electronic Coulomb interaction on the spin-orbit-coupling induced spin Hall effect. We have shown that the electronic Coulomb interaction may substantially decrease the spinorbit-coupling induced transverse spin accumulation in a sample, and the decrease of the transverse spin accumulation due to the influences of the Coulomb interaction is determined by the ratio of the spin-drag resistivity to the ordinary resistivity of a sample. In order to get a proper estimate of the spin Hall effect in real materials, these influences may need to be taken into account.

This work was supported by a grant from the Research Grant Council of Hong Kong, China.
${ }^{1}$ J.E. Hirsch, Phys. Rev. Lett. 83, 1834 (1999).

${ }^{2}$ S. Zhang, Phys. Rev. Lett. 85, 393 (2000).

${ }^{3}$ M. Johnson and R.H. Silsbee, Phys. Rev. Lett. 55, 1790 (1985); M. Johnson, ibid. 70, 2142 (1993).

${ }^{4}$ T. Valet and A. Fert, Phys. Rev. B 48, 7099 (1993).

${ }^{5}$ W.P. Pratt, Jr., S.F. Lee, J.M. Slaughter, R. Loloee, P.A. Schroeder, and J. Bass, Phys. Rev. Lett. 66, 3060 (1991).

${ }^{6}$ M.A.M. Gijs, S.K.J. Lenczowski, and J.B. Giesbers, Phys. Rev. Lett. 70, 3343 (1993).

${ }^{7}$ C. M. Hurd, The Hall Effects in Metals and Alloys (Plenum, New York, 1973), Chap. 5, and references therein.
${ }^{8}$ C. L. Chien and C. R. Westgate, The Hall Effects and Its Applications (Plenum Press, New York, 1980).

${ }^{9}$ S.A. Wolf et al., Science 294, 1488 (2001); G.A. Prinz, Phys. Today 48(4), 58 (1995).

${ }^{10}$ S. Takahashi and S. Maekawa, Phys. Rev. Lett. 88, 116601 (2002).

${ }^{11}$ I. D'Amico and G. Vignale, Phys. Rev. B 62, 4853 (2000); 65, 085109 (2001).

${ }^{12}$ S. Zhang, Phys. Rev. B 51, 3632 (1995).

${ }^{13}$ E.N. Adams and E.I. Blount, J. Phys. Chem. Solids 10, 286 (1959). 